\title{
Tailoring single-cycle near field in a tunnel junction with carrier-envelope phase-controlled terahertz
}

\section{electric fields}

Katsumasa Yoshioka ${ }^{l}$, Ikufumi Katayama ${ }^{l^{*}}$, Yusuke Arashida ${ }^{l}$, Atsuhiko Ban ${ }^{l}$, Yoichi Kawada ${ }^{2}$, Kuniaki Konishi ${ }^{3}$, Hironori Takahashi ${ }^{2}$, and Jun Takeda ${ }^{{ }^{*}}$

${ }^{1}$ Department of Physics, Graduate School of Engineering, Yokohama National University, Yokohama 240-8501, Japan

${ }^{2}$ Central Research Laboratory, Hamamatsu Photonics K.K., 5000 Hirakuchi, Hamakita, Hamamatsu City, Shizuoka 434-8601, Japan

${ }^{3}$ Institute for Photon Science and Technology, Graduate School of Science, The University of Tokyo, Tokyo 113-0033, Japan

KEYWORDS: THz-STM, single-cycle near field, light-field-driven process, ultrafast and ultrasmall 
ABSTRACT: Light-field-driven processes occurring under conditions far beyond the diffraction limit of the light can be manipulated by harnessing spatio-temporally tunable near fields. A tailor-made carrier envelope phase in a tunnel junction formed between nanogap electrodes allows precisely controlled manipulation of these processes. In particular, the characterization and active control of near fields in a tunnel junction are essential for advancing elaborate manipulation of light-field-driven processes at the atomic-scale. Here, we demonstrate that desirable phase-controlled near fields can be produced in a tunnel junction via terahertz scanning tunneling microscopy (THz-STM) with a phase shifter. Measurements of the phase-resolved subcycle electron tunneling dynamics revealed an unexpected large carrier-envelope phase shift between far-field and near-field single-cycle $\mathrm{THz}$ waveforms. The phase shift stems from the wavelength-scale feature of the tip-sample configuration. By using a dual-phase double-pulse scheme, the electron tunneling was coherently manipulated over the femtosecond timescale. Our new prescription - in situ tailoring of single-cycle THz near fields in a tunnel junction — will offer unprecedented control of electrons for ultrafast atomic-scale electronics and metrology. 
TEXT: Strong-field interactions of light with matter have recently attracted significant attention [1-9]. The strong field produced by few-cycle ultrashort laser pulses can control the motion of electrons in the non-perturbative regime. In this regime, precise control of the carrier-envelope phase (CEP) of ultrashort laser pulses enabled the: steering of light-field-driven currents in solidstate systems [1,2], high-harmonic generation of attosecond pulses [3], and the chemical reaction of molecules $[4,5]$ at the sub-cycle timescale. Focusing of CEP-controlled ultrashort laser pulses beyond the diffraction limit will provide fascinating avenues for manipulating light-field-driven processes in the ultrafast and ultrasmall regime. The development of strong-field physics has been greatly facilitated by locally enhanced near fields in nanostructures [10-18]. Near-fieldmediated electron manipulations with the CEP-controlled laser pulses have been demonstrated for either electron emission from metal nanotips $[10,11]$ or dielectric nanospheres $[12,13]$. The use of nanostructures provides the large-field enhancement and nanoscale confinement of light beyond the diffraction limit. However, owing to their geometry, the laser fields employed in these studies were limited to inducing photoemission from materials.

Light-field-driven quantum tunneling between nanogap electrodes using phase-locked single-cycle pulses has recently emerged as a new method for ultrafast coherent control of electrons at the nanoscale. CEP-dependent electron tunneling in near-infrared (NIR) [19] and terahertz $(\mathrm{THz})[20]$ spectral ranges as well as atomic-resolution imaging of ultrafast dynamics via terahertz scanning tunneling microscopy (THz-STM) [21,22] have been reported. Conventional STM is applicable to any type of conducting material, such as superconductors [23], magnetic materials [24], and two-dimensional nanomaterials [25]. Therefore, THz-STM with CEP-controlled single-cycle $\mathrm{THz}$ pulses will pave the way for a new phase of ultrafast atomic-scale investigations; for example, spatiotemporal manipulations of phase transitions 
[26,27], coherent spin dynamics [28], and chemical reactions are expected [29]. Precise characterization and active control of near fields in a tunnel junction with sub-cycle resolution are therefore crucial for advancing these next-generation light-field-driven manipulations. Streaking spectroscopy, originally developed for atomic systems and commonly used in attosecond science [30,31], constitutes a promising means of visualizing optical near fields in nanostructures. In fact, for both $\mathrm{THz}$ [15] and NIR [16] pulses, this technique has been successfully applied to the retrieval of optical near fields at the tip apex of a nanoscale metal tip. This retrieval relied on a dual-wavelength double-pulse scheme with different pulse durations and ponderomotive energies. The photoemitted electrons initiated by a pulse with a relatively short wavelength and pulse duration were accelerated by the near field of a long wavelength phase-locked pulse with large ponderomotive energy. However, streaking spectroscopy is inapplicable to a tunnel junction as the electron transfer occurs only between nanogap electrodes via quantum tunneling activated by the electric field of light. Electron removal from the junction is therefore impossible. An experimental method for reconstructing single-cycle near fields remains elusive and, hence, previous studies [20-22] have assumed that $\mathrm{THz}$ far fields and near fields in a tunnel junction are identical.

In the present study, we demonstrate the sub-cycle retrieval of $\mathrm{THz}$ near fields in a tunnel junction by using a combination of THz-STM and a CEP shifter [32]. Regardless of the nanotip shape, we could implement in situ tailoring of single-cycle THz near fields in the junction. We also achieved precise control of the electron tunneling over the femtosecond timescale by employing a Mach-Zehnder interferometer with a dual-phase double-pulse scheme. 
The basic concept of our experimental set-up is illustrated in Fig. 1a. Highly oriented pyrolytic graphite (HOPG) was used as a sample, owing to its atomically flat surface. Singlecycle THz electric fields were generated via optical rectification of NIR femtosecond pulses in a LiNbO3 prism using a tilted-pulse-front configuration [33]. The generated $\mathrm{THz}$ pulses were then

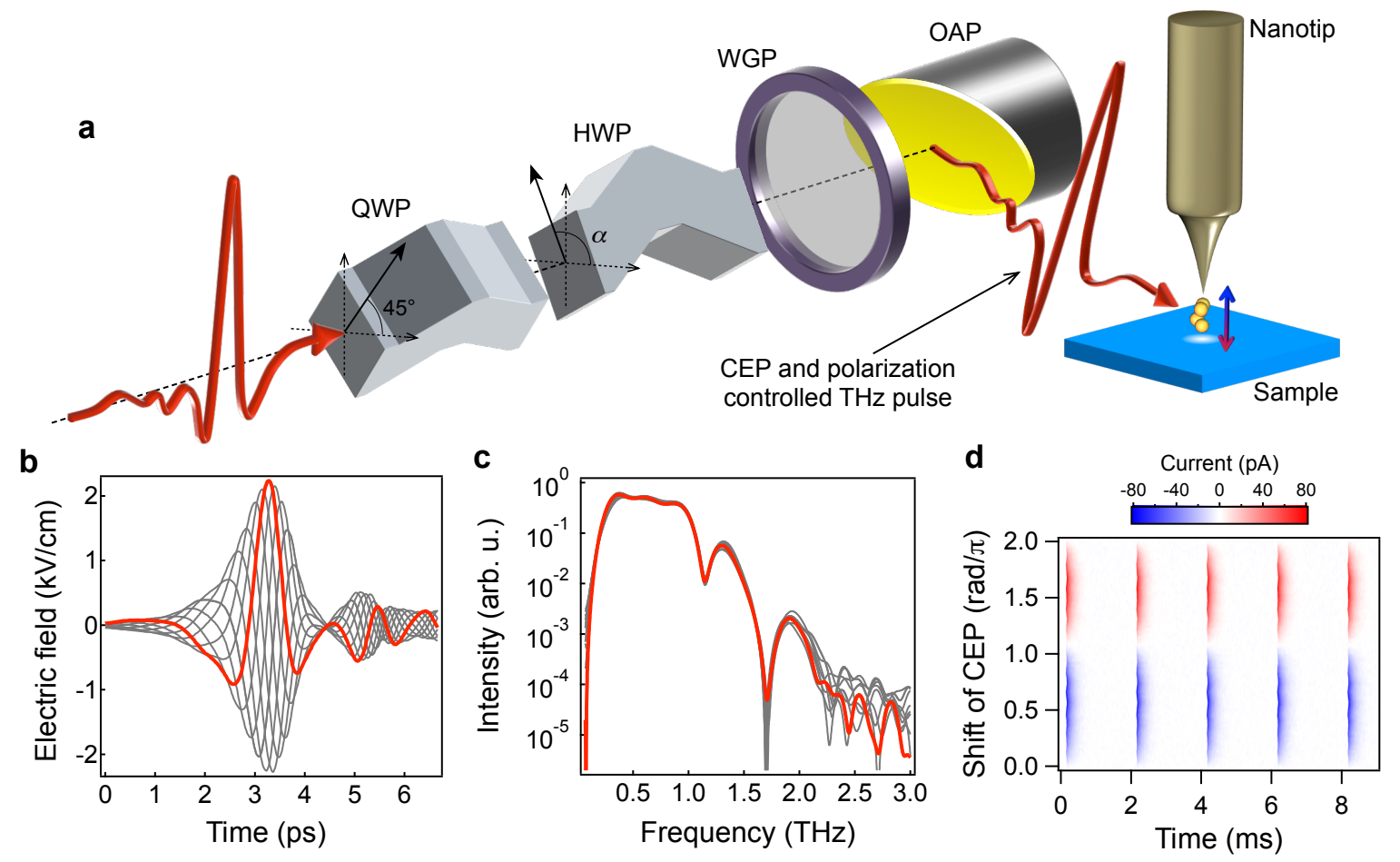

Figure 1. (a) Schematic of the experimental setup. QWP: quarter-wave plate, HWP: halfwave plate, WGP: wire grid polariser, OAP: off-axis parabolic mirror. CEP-and polarisationcontrolled single-cycle THz electric fields are focused onto the apex of a Pt/Ir nanotip with an incident angle of $75^{\circ}$. (b) Far-field single-cycle $\mathrm{THz}$ waveforms with different CEPs measured via electro-optic sampling (EOS). The dotted grey lines show the CEP-controlled waveforms for each (2/9) $\pi$ shift. (c) THz frequency spectra obtained through Fourier transformation of (b). (d) THz-driven tunnel currents as a function of the CEP shift of a THz electric field without a direct current (d.c.) bias. The feedback loop remained off during the measurement (setpoint: bias voltage $V_{\mathrm{s}}=1 \mathrm{~V}$, tunnel current $I_{\mathrm{s}}=0.1 \mathrm{nA}$ ). 
guided into a CEP shifter [32], which was composed of three optical elements (a quarter-wave plate, half-wave plate, and wire-grid polarizer) for broadband THz pulses [34]. The CEP of the THz pulses that pass through the CEP shifter is given by $\phi_{\text {CEP }}=\phi_{\text {CEPO }}+2 \alpha$, where $\phi_{\text {CEP }}$ and $\phi_{\text {CEPO }}$ are the output and initial CEPs, respectively, and $\alpha$ is the azimuth angle of the half-wave plate. CEP adjustment either through the insertion of a pair of fused-silica wedges $[1,2,4,11,12,19]$ or spherical and cylindrical lenses [20] leads to variations in the time delay, pulse duration, and frequency component. However, the CEP shifter enables precise control of the CEP with values that change continuously from $0-2 \pi$ without the occurrence of these variations, as shown in Fig. $1 \mathrm{~b}$ and $\mathrm{c}$. The CEP of single-cycle THz pulses tuned by the CEP shifter is highly stable, owing to the robustness of our optical setup and the steady $\phi_{\text {CEP } 0}$ value passively locked through the optical rectification. Furthermore, the CEP-controlled single-cycle $\mathrm{THz}$ pulses were focused onto the apex of a Pt/Ir nanotip. By irradiating a tunnel junction with these pulses while continuously tuning the CEP by $2 \pi$, we could observe a series of CEP-dependent tunnel currents using an oscilloscope (see Fig. 1d). The time interval of each current pulse $(2 \mathrm{~ms})$ is equal to the repetition rate of the irradiated pulses (see Supporting Section I). The time-integrated value of each current pulse at a given CEP represents the number of electrons driven by a phase-controlled singlecycle THz pulse through the junction. 
Figure 2a shows the number of electrons as a function of CEP with different $\mathrm{THz}$ pulse energies. The direction and number of electrons tunneling through the junction are strongly dependent on the CEP. The number of rectified electrons is maximized at $0.67 \pi$ for electrons tunneling from the nanotip to the sample and at $1.67 \pi$ for the opposite scenario. In these cases, the $\mathrm{THz}$ waveform in the junction is expected to be a single-cycle co-sinusoidal near field; the near field with either positive or negative polarity induces the unidirectional tunnel current, indicating the symmetry breaking of electric transport. In contrast, the number of rectified electrons is minimized at $0.06 \pi, 1.17 \pi$ and $2.11 \pi$, corresponding to a sinusoidal near field in the junction; the near field with symmetric positive and negative polarities drives the bidirectional
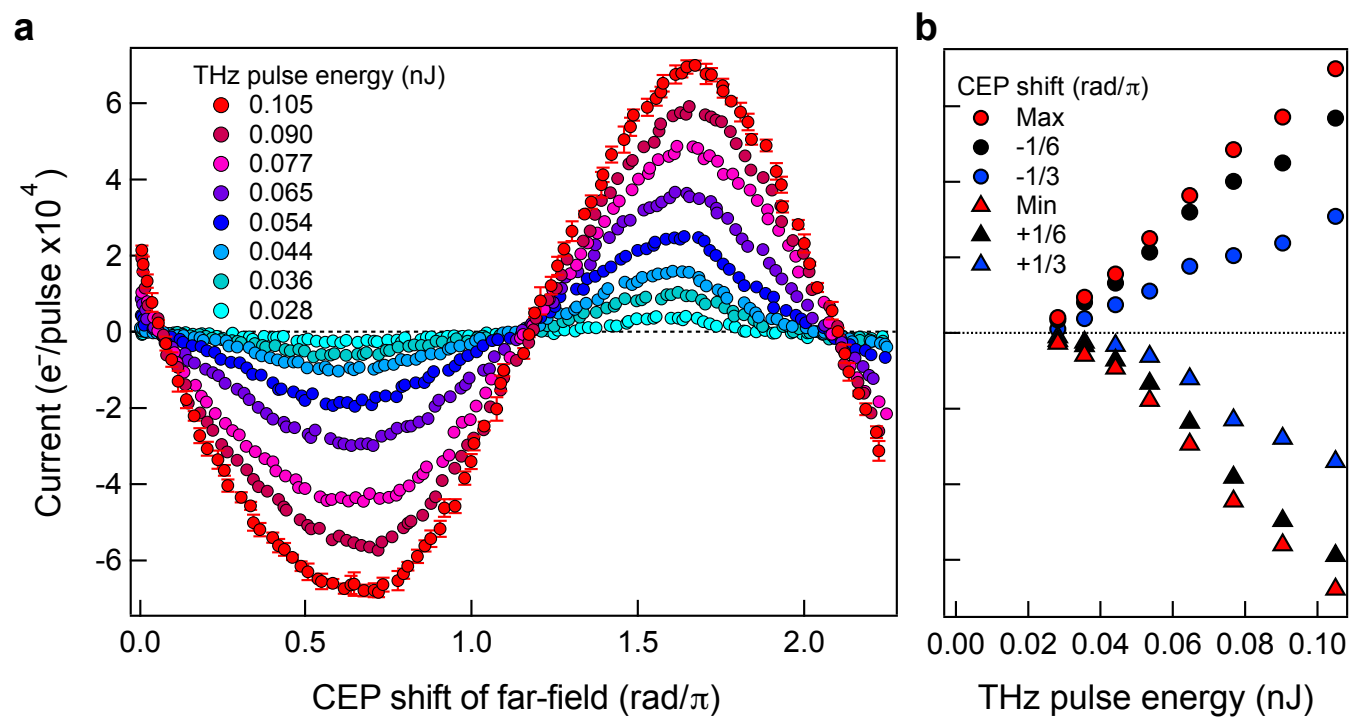

Figure 2. (a) Number of electrons driven by a single THz pulse as a function of the CEP with different THz pulse energies $\left(V_{\mathrm{s}}=1 \mathrm{~V}, I_{\mathrm{s}}=0.1 \mathrm{nA}\right)$. The electrons undergo tunneling from the nanotip to the sample with positive values, and vice versa for negative values. (b) Increase in the number of electrons with $\mathrm{THz}$ pulse energy associated with (a). The $\mathrm{THz}$ pulse energy was calculated from the corresponding $\mathrm{THz}$ waveform by integrating the waveform intensity both temporally and spatially. 
tunnel current with the same number of electrons. An increase in the number of electrons with THz pulse energy exhibits threshold-like non-linear behavior, as shown in Fig. 2b, owing to the strong modulation of the potential barrier in the junction [20]. This CEP-dependent non-linear increase in the electron tunneling indicates that the electron transfer originates from the THzfield-driven process. Therefore, the tunneling current exhibits a strong dependence on the CEP of THz near fields.

We investigated the $\mathrm{THz}$ near fields in the junction by comparing with their far-field waveforms. These waveforms were experimentally obtained via electro-optic sampling (EOS), and the number of electrons driven by the far fields was calculated using the Simmons model [35]. During these calculations, the THz-field-induced sub-cycle modulation of a potential barrier with an image potential was taken into consideration. The values of the effective work function and gap width $(3.8 \mathrm{eV}$ and $1.0 \mathrm{~nm}$, respectively) used in the calculation were both obtained from current-distance (I-Z) experiments with a direct current (dc) electric field. Here, the $\mathrm{THz}$ electric field was treated as a quasi-static field because the I-V response in the $\mathrm{THz}$ regime is the same as that in the dc regime [20] (see Supplementary Information III in [20]). The results for three different nanotips (Tip 1, Tip 3 and Tip 5, see Fig. 3b) are shown in Fig. 3a. As the figure shows, the CEP dependence of electrons rectified by the single-cycle THz near field of each nanotip differs completely from that rectified by the corresponding single-cycle $\mathrm{THz}$ far field. This dependence varies significantly with the shape of the nanotips. Measurements revealed a maximum CEP shift of $\pi / 2$ between the near and far fields; this shift was ignored in previous studies [20-22]. The result for five different nanotips is summarized in Fig. 3c. The large CEP shift can be attributed to the antenna properties induced by local fields at the apex of a nanotip $[15,36,37]$. We simulated the THz near field in the junction by using an antenna model 


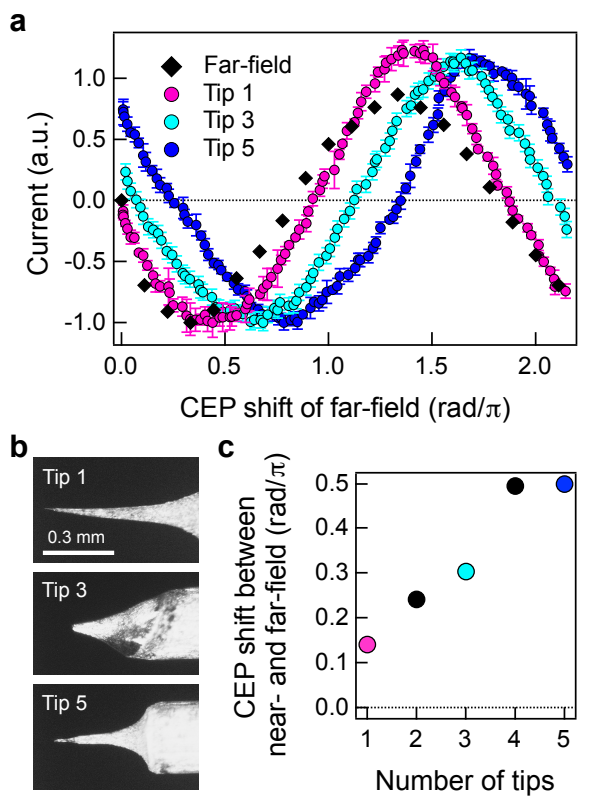

Figure 3. (a) CEP-resolved rectified electrons with different nanotips $\left(V_{\mathrm{s}}=1 \mathrm{~V}, I_{\mathrm{s}}=0.1\right.$ nA). The CEP-resolved rectified electrons induced by the corresponding far field, which is calculated using the Simmons model, is also shown. (b) Optical micrographs of the electrochemically etched Pt/Ir nanotips used in a. (c) CEP shift of each THz near field for five different nanotips. The coloured symbols correspond to the data shown in a.

that assigns an equivalent RLC circuit to the nanotip. As Fig. 4a shows, the results correspond closely to the experimental data, which were calculated using the corresponding far-field waveform and adjustable circuit parameters depending on the nanotip (see Supporting Section II). The difference in the CEP shift of the nanotips originates from the difference in resonance frequency and resistance which depend primarily on the overall shape of the nanotip. Although the far-field waveform is identical among different nanotips, the resulting $\mathrm{THz}$ near field and subsequent electron current depend strongly on the nanotip (Fig. 4b). Reproduction of the enhanced near-field waveform in the junction is difficult and requires more than simply multiplying the corresponding far-field waveform by each field enhancement factor. 
Consequently, the direction of electron tunneling depends on the nanotip. In the case of Tip 1, the small CEP shift leads to electron transfer in the same direction as that expected for the farfield waveform. In the case of Tip 5, however, the transfer occurs in the opposite direction due to the large CEP shift. In the case of Tip 3, electrons flow equally in each direction, owing to the sinusoidal-shaped near field with a moderate CEP shift.

For a thorough understanding of the tip-dependent CEP shift as shown in Fig. 3c, we
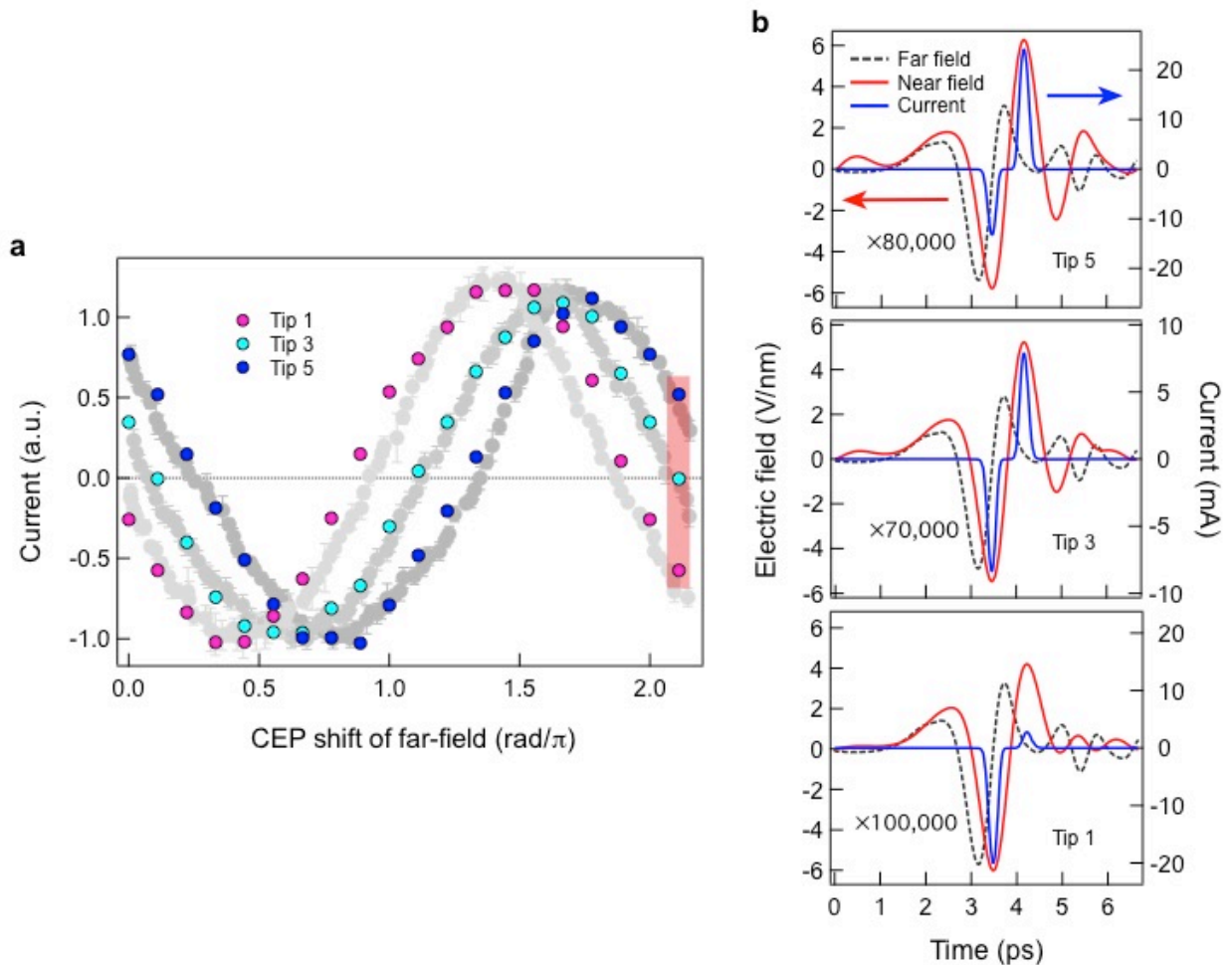

Figure 4. (a) CEP-resolved rectified electrons calculated from the antenna model. The simulation parameters are summarised in Supporting Section II. The grey circles denote the measured CEP-resolved rectified electrons (corresponding to Fig. 3a). (b) Tip dependence of far-field and near-field waveforms, and that of the resulting tunnel currents, at a CEP shift of $2.1 \pi$ rad (highlighted by the red rectangle in (a)). For comparison with the near field, the far field was magnified by field enhancement factors ranging from 70,000 to $100,000$. 
performed the finite integration simulation of the $\mathrm{THz}$ near-field waveform in a tunnel junction using a commercially available software, CST MW STUDIO (see Supporting Section III for more details). Figure 5a shows the tip-sample configuration used in the simulation to investigate the effect of the nanotip macroscopic feature. The tip shape was assumed to be a Lorentzian with different heights. We confirmed that the waveform of local electric field was insensitive to gap distances ranging from $1.0 \mathrm{~nm}$ to $10 \mu \mathrm{m}$ (see Supporting Section III for more details). Therefore, the gap distance between the tip and a sample was set to $10 \mu \mathrm{m}$. We used an actual THz far-field waveform measured via EO sampling, which was incident on the tip at $75^{\circ}$. As shown in Fig. 5b, the THz near-field waveform depends strongly on the tip shape, indicating that the tip-dependent CEP shift observed in the experiment results from the macroscopic shape of the tip. The tip with lower height is characterized by a co-sinusoidal shape, while the tip with higher height is characterized by a sinusoidal shape. The estimated CEP shift between the far and near fields is summarized in Fig. $5 c$. The CEP shift increases up to $0.47 \pi$ with tip height, consistent with the observed range of the CEP shift. Roughly speaking, the tip with lower height behaves only as a scattered medium having almost no phase shift. However, the tip with the wavelength-scale height behaves as an antenna and therefore yield a large phase shift of $\sim 0.5 \pi$. This indicates that the antenna effect becomes weaker when the tip geometry becomes small compared to the incident $\mathrm{THz}$ wavelength. 
Our results demonstrate that although the electron transfer occurs on the nanoscale via a tunnel junction, the spatiotemporal behavior of this transfer is determined by the overall shape of the nanotip. This behavior results from the relatively long wavelength of the $\mathrm{THz}$ electric field. In actual experiments, control of the tip shape - tip height, curvature and symmetry - is very difficult. Therefore, determining the CEP of a THz near field from the actual tip shape prior to the THz-STM experiment (for example, see Fig. 3b and c) is impossible. However, our scheme for THz-STM with a CEP shifter enables in situ tailoring of the CEP-controlled single-cycle THz near field with sub-cycle resolution. This prescription for retrieving the $\mathrm{THz}$ near field in a tunnel junction holds significant promise for unprecedented control of electrons in ultrafast
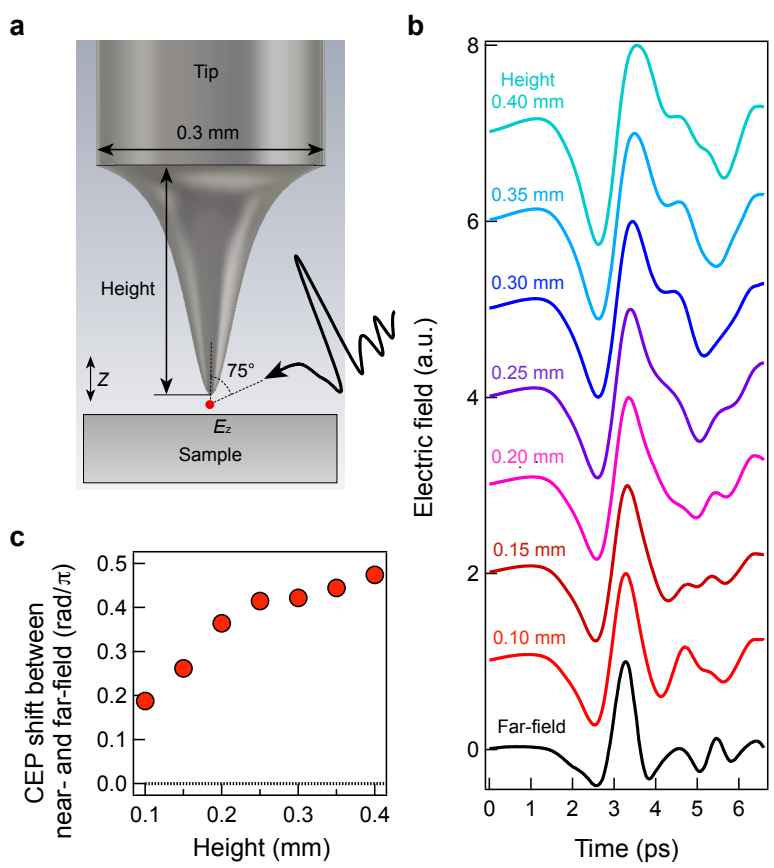

Figure 5. (a) Tip-sample configuration with a gap width of $10 \mu \mathrm{m}$. The waveform of far-field THz electric fields was measured via electro-optic sampling (Fig. 1b). (b) Far- and near-field waveforms with different tip heights. For clarity, each waveform was normalized at the maximum peak electric field and offset by unity. (c) CEP shift between each THz near field and far field as a function of the tip height. 
atomic-scale electronics and metrology.

To reveal ultrafast dynamics of near-field-mediated electron tunneling with sub-cycle time resolution, we employed a Mach-Zehnder interferometer, with a dual-phase double-pulse scheme. This interferometer has an additional delay-controlled $\mathrm{THz}$ pulse with a fixed CEP (see Supporting Section I), as schematically illustrated in Fig. 6a. The timing and direction of the electron tunneling were controlled and observed over a femtosecond timescale, as shown in Fig.

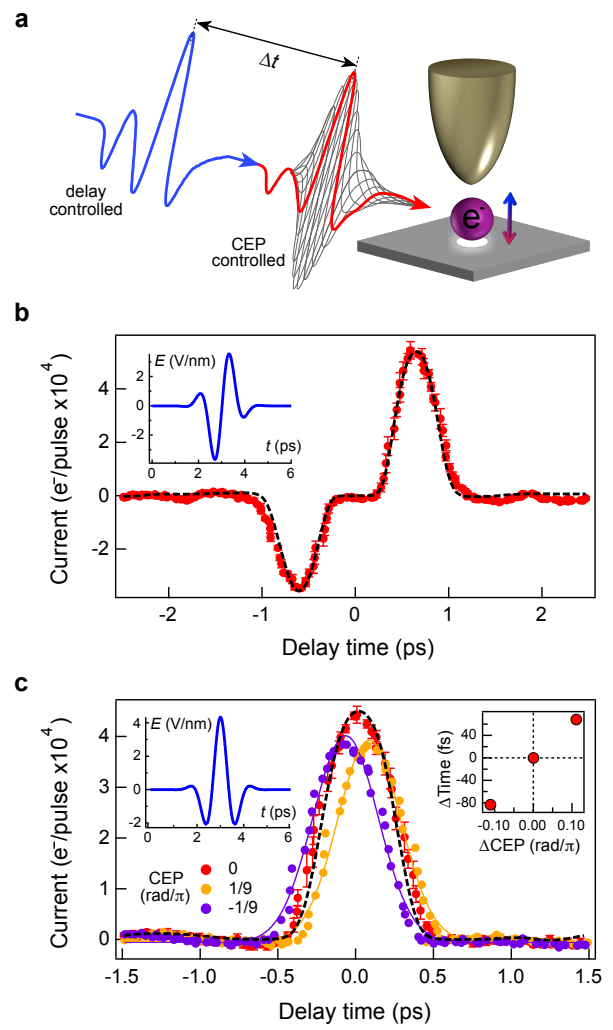

Figure 6. (a) Schematic of the experimental concept. An additional single-cycle $\mathrm{THz}$ pulse with a fixed CEP was used with a given delay time from a CEP-controlled single-cycle THz pulse. (b), (c) Number of electrons as a function of the delay time with sinusoidal (b) and cosinusoidal near fields (c) as shown in the inset waveforms $\left(V_{\mathrm{s}}=1 \mathrm{~V}, I_{\mathrm{s}}=0.1 \mathrm{nA}\right)$. The dashed curves show the best fit obtained by the Simmons model. The inset in (c) shows the temporal shift of the current burst resulting from CEP tuning. 
$6 \mathrm{~b}$ and c. Furthermore, the CEP-controlled first pulse was adjusted to yield either a sinusoidal (Fig. 6b) or co-sinusoidal (Fig. 6c) near field, as shown in the insets. In the case of the (i) sinusoidal near field, a bidirectional sub-picosecond electron burst was generated with an interval of $1.2 \mathrm{ps}$ and (ii) co-sinusoidal near field, a unidirectional electron burst was produced with a pulse duration (full width at half maximum) of $490 \mathrm{fs}$. These electron bursts are accurately reproduced (as indicated by the dashed lines in Fig. $6 \mathrm{~b}$ and c) by the Simmons model (corresponding waveform shown in the insets). This ultrafast electron burst is highly sensitive to the CEP. As shown in the right inset of Fig. 6c, the peak position of the electron burst is shifted by $70 \mathrm{fs}$ and $-80 \mathrm{fs}$ for CEP shifts of $(1 / 9) \pi$ and $-(1 / 9) \pi$, respectively. This indicates that our method can also manipulate the near-field-mediated electron dynamics in a tunnel junction with femtosecond time resolution. In fact, this precise active control of both the near-field waveform and the resulting electron tunneling is unattainable without our stable and robust THz-STM equipped with a CEP shifter. This shifter can control the desirable CEP for THz near fields without additional delay and chirp. We believe that our double-pulse scheme with tailor-made THz near fields will provide unique capability for future sub-cycle time-resolved experiments in a tunnel junction, for example, by selectively accessing specific energy levels such as HOMO and LUMO states in molecules [21] and superconducting gap in superconductors [23].

Finally, we will discuss the difference between scattering-type near-field scanning optical microscopy (s-NSOM) and our CEP-controlled THz-STM based on their operating principles: sNSOM measures a local electric field underneath the nanotip, while THz-STM measures a tunnel current at a tunnel junction. In the case of s-NSOM [36-41], the near-field signal is extracted from a relatively high far-field background by nanotip tapping and higher harmonic demodulations. In contrast, our CEP-controlled THz-STM can retrieve a near-field waveform via 
sub-cycle electron tunneling spectroscopy without isolation of the near field from its far field, because of a large enhancement factor of the near field in a tunnel junction $(\sim 100,000)$ $[20,22,42]$. Based on the operating principles, the spatial resolution of THz-STM is much higher than that of s-NSOM. The spatial resolution of s-NSOM is the order of $10 \mathrm{~nm}[38,39]$, while that of our THz-STM is around 1-2 nm even under ambient laboratory condition [43]; it can be further reduced to $0.06 \mathrm{~nm}$ by using low-temperature and ultrahigh-vacuum STM [21]. The limitations of THz-STM are as follows. First, the sample surface should be electrically conductive otherwise we cannot measure the tunnel current. Second, I-V curve in the junction should exhibit nonlinear behavior with respect to the $\mathrm{THz}$ electric field because the operation of THz-STM relies on a nonlinear rectification process in the junction [20,44].

In summary, we have demonstrated the characterization and active control of $\mathrm{THz}$ near fields in a tunnel junction by precisely adjusting the CEP of a single-cycle $\mathrm{THz}$ electric field. Measurements of the CEP-resolved sub-cycle tunneling dynamics revealed a large CEP shift between single-cycle $\mathrm{THz}$ far and near fields. Based on the antenna model that assigns an equivalent RLC circuit to the nanotip, we could precisely retrieve the $\mathrm{THz}$ near field, regardless of the shape of the nanotips. Using the finite integration simulation, we found that the CEP shift is determined by the wavelength-scale feature of the tip-sample configuration. Use of the activecontrolled double pulses enabled coherent control of the electron transfer in the junction on the femtosecond timescale. Moreover, we could create a desirable near field in the junction, even if the CEP shift between far and near fields resulting from the tip-sample configuration was unpredictable. Our method is applicable to the $\mathrm{THz}$ spectral range and considerably higherfrequency ranges, such as the visible range $[45,46]$. In these higher-frequency ranges, we expect a higher sensitivity of the near-field waveform for smaller geometrical shapes of the nanotips 
(than for larger shapes). In addition, the dispersion of the dielectric function and the penetration depth into the junction are expected to be non-negligible. The use of artificially-made structures for investigating the adiabatic focusing of surface plasmons would also be interesting $[47,48]$. We believe that the concept presented here facilitates the development of strong-field physics and next-generation ultrafast atomic-scale electronics and metrology, which are the building blocks of future information technology.

\section{ASSOCIATED CONTENT}

\section{Supporting Information}

The Supporting Information is available free of charge on the ACS Publications website at DOI: .

Description of the experimental setup for the THz-STM. Explanation of near field calculation using the antenna model. Details of electromagnetic simulation for $\mathrm{THz}$ near fields at a single tunnel junction (PDF).

\section{AUTHOR INFORMATION}

\section{Corresponding Authors}

*E-mail: jun@ynu.ac.jp; katayama@ynu.ac.jp.

\section{Author Contributions}


K.Y., I.K. and J.T. conceived and coordinated this project. K.Y. designed and built the THzSTM setup with support from A.B., K.Y. and Y.K. and H.T. constructed the operating system of the STM with a phase shifter. K.Y. performed the experiments and simulations with support from A.B., I.K. and Y.A. Y.A. and K.K. conducted the numerical simulation based on the finite integration technique. K.Y. and J.T. wrote this manuscript with contribution from all authors.

\section{Notes}

The authors declare no competing financial interest.

\section{ACKNOWLEDGMENT}

This work was supported in part by the Grants-in-Aid for Scientific Research (Nos. 16H04001, 16H06010 and 18H04288) and Grant-in-Aid for JSPS Research Fellow (17J05234) from the Japan Society for the Promotion of Science and the Ministry of Education, Culture, Sports, Science and Technology. Grant-in-Aid from the Strategic Information and Communications

R\&D Promotion Programme (SCOPE \#145003103) of the Japan Ministry of Internal Affairs and Communications is also gratefully acknowledged. The authors would also like to thank Dr. Takayuki Kurihara for fruitful discussions. 


\section{REFERENCES}

(1) Schiffrin, A.; Paasch-Colberg, T.; Karpowicz, N.; Apalkov, V.; Gerster, D.; Mühlbrandt,

S.; Korbman, M.; Reichert, J.; Schultze, M.; Holzner, S.; Barth, J. V; Kienberger, R.; Ernstorfer, R.; Yakovlev, V. S.; Stockman, M. I.; Krausz, F. Nature 2012, 493, 70-74.

(2) Higuchi, T.; Heide, C.; Ullmann, K.; Weber, H. B.; Hommelhoff, P. Nature 2017, 550, 224-228.

(3) Baltuška, A.; Udem, T.; Uiberacker, M.; Hentschel, M.; Goulielmakis, E.; Gohle, C.; Holzwarth, R.; Yakovlev, V. S.; Scrinzi, A.; Hänsch, T. W.; Krausz, F. Nature 2003, 421, 611615.

(4) Kling, M. F.; Siedschlag, Ch.; Verhoef, A. J.; Khan, J. I.; Schultze, M.; Uphues, Th.; Uiberacker, Y. N. M.; Drescher, M.; Krausz, F.; Vrakking, M. J. J. Science 2006, 312, 246-248.

(5) Kübel, M.; Siemering, R.; Burger, C.; Kling, N. G.; Li, H.; Alnaser, A. S.; Bergues, B.; Zherebtsov, S.; Azzeer, A. M.; Ben-Itzhak, I.; Moshammer, R.; de Vivie-Riedle, R.; Kling, M. F. Phys. Rev. Lett. 2016, 116, 193001.

(6) Schultze, M.; Bothschafter, E. M.; Sommer, A.; Holzner, S.; Schweinberger, W.; Fiess, M.; Hofstetter, M.; Kienberger, R.; Apalkov, V.; Yakovlev, V. S.; Stockman, M. I.; Krausz, F. Nature 2013, 493, 75-78.

(7) Luu, T. T.; Garg, M.; Kruchinin, S. Y.; Moulet, A.; Hassan, M. T.; Goulielmakis, E. Nature 2015, 521, 498-502.

(8) Hohenleutner, M.; Langer, F.; Schubert, O.; Knorr, M.; Huttner, U.; Koch, S. W.; Kira, M.; Huber, R. Nature 2015, 523, 572-575. 
(9) Langer, F.; Hohenleutner, M.; Schmid, C. P.; Poellmann, C.; Nagler, P.; Korn, T.; Schüller, C.; Sherwin, M. S.; Huttner, U.; Steiner, J. T.; Koch, S. W.; Kira, M.; Huber, R. Nature 2016, $533,225-229$.

(10) Krüger, M.; Schenk, M.; Hommelhoff, P. Nature 2011, 475, 78-81.

(11) Piglosiewicz, B.; Schmidt, S.; Park, D. J.; Vogelsang, J.; Groß, P.; Manzoni, C.; Farinello, P.; Cerullo, G.; Lienau, C. Nat. Photonics 2014, 8, 37-42.

(12) Zherebtsov, S.; Fennel, T.; Plenge, J.; Antonsson, E.; Znakovskaya, I.; Wirth, A.; Herrwerth, O.; Süßmann, F.; Peltz, C.; Ahmad, I.; Trushin, S. A.; Pervak, V.; Karsch, S.; Vrakking, M. J. J.; Langer, B.; Graf, C.; Stockman, M. I.; Krausz, F.; Rühl, E.; Kling, M. F. Nat. Phys. 2011, 7, 656-662.

(13) Süßmann, F.; Seiffert, L.; Zherebtsov, S.; Mondes, V.; Stierle, J.; Arbeiter, M.; Plenge, J.; Rupp, P.; Peltz, C.; Kessel, a.; Trushin, S. a.; Ahn, B.; Kim, D.; Graf, C.; Rühl, E.; Kling, M. F.; Fennel, T. Nat. Commun. 2015, 6, 7944.

(14) Herink, G.; Solli, D. R.; Gulde, M.; Ropers, C. Nature 2012, 483, 190-193.

(15) Wimmer, L.; Herink, G.; Solli, D. R.; Yalunin, S. V.; Echternkamp, K. E.; Ropers, C. Nat. Phys. 2014, 10, 432-436.

(16) Förg, B.; Schötz, J.; Süßmann, F.; Förster, M.; Krüger, M.; Ahn, B.; Okell, W. A.; Wintersperger, K.; Zherebtsov, S.; Guggenmos, A.; Pervak, V.; Kessel, A.; Trushin, S. A.; Azzeer, A. M.; Stockman, M. I.; Kim, D.; Krausz, F.; Hommelhoff, P.; Kling, M. F. Nat. Commun. 2016, 7, 11717. 
(17) Hoff, D.; Krüger, M.; Maisenbacher, L.; Sayler, A. M.; Paulus, G. G.; Hommelhoff, P. Nat. Phys. 2017, 13, 947-951.

(18) Vampa, G.; Ghamsari, B. G.; Siadat Mousavi, S.; Hammond, T. J.; Olivieri, A.; LisickaSkrek, E.; Naumov, A. Y.; Villeneuve, D. M.; Staudte, A.; Berini, P.; Corkum, P. B. Nat. Phys. 2017, 13, 659-662.

(19) Rybka, T.; Ludwig, M.; Schmalz, M. F.; Knittel, V.; Brida, D.; Leitenstorfer, A. Nat. Photonics 2016, 10, 667-670.

(20) Yoshioka, K.; Katayama, I.; Minami, Y.; Kitajima, M.; Yoshida, S.; Shigekawa, H.; Takeda, J. Nat. Photonics 2016, 10, 762-765.

(21) Cocker, T. L.; Peller, D.; Yu, P.; Repp, J.; Huber, R. Nature 2016, 539, 263-267.

(22) Jelic, V.; Iwaszczuk, K.; Nguyen, P. H.; Rathje, C.; Hornig, G. J.; Sharum, H. M.; Hoffman, J. R.; Freeman, M. R.; Hegmann, F. A. Nat. Phys. 2017, 13, 591-598.

(23) Hanaguri, T.; Niitaka, S.; Kuroki, K.; Takagi, H. Science 2010, 328, 474-476.

(24) Wachowiak, A.; Wiebe, J.; Bode, M.; Pietzch, O.; Morgenstern, M.; Wiesendanger, R. Science 2002, 298, 577-580.

(25) Tapasztó, L.; Dobrik, G.; Lambin, P.; Biró, L. P. Nat. Nanotechnol. 2008, 3, 397-401.

(26) Liu, M.; Hwang, H. Y.; Tao, H.; Strikwerda, A. C.; Fan, K.; Keiser, G. R.; Sternbach, A. J.; West, K. G.; Kittiwatanakul, S.; Lu, J.; Wolf, S. a; Omenetto, F. G.; Zhang, X.; Nelson, K. a; Averitt, R. D. Nature 2012, 487, 345-348. 
(27) Zalden, P.; Shu, M. J.; Chen, F.; Wu, X.; Zhu, Y.; Wen, H.; Johnston, S.; Shen, Z.-X.; Landreman, P.; Brongersma, M.; Fong, S. W.; Wong, H.-S. P.; Sher, M.; Jost, P.; Kaes, M.; Salinga, M.; von Hoegen, A.; Wuttig, M.; Lindenberg, A. M. Phys. Rev. Lett. 2016, 117, 067601.

(28) Kubacka, T.; Johnson, J. A.; Hoffmann, M. C.; Vicario, C.; de Jong, S.; Beaud, P.; Grubel, S.; Huang, S.-W.; Huber, L.; Patthey, L.; Chuang, Y.-D.; Turner, J. J.; Dakovski, G. L.; Lee, W.S.; Minitti, M. P.; Schlotter, W.; Moore, R. G.; Hauri, C. P.; Koohpayeh, S. M.; Scagnoli, V.; Ingold, G.; Johnson, S. L.; Staub, U. Science. 2014, 343, 1333-1336.

(29) LaRue, J. L.; Katayama, T.; Lindenberg, A.; Fisher, A. S.; Öström, H.; Nilsson, A.; Ogasawara, H. Phys. Rev. Lett. 2015, 115, 036103.

(30) Itatani, J.; Quéré, F.; Yudin, G. L.; Ivanov, M. Y.; Krausz, F.; Corkum, P. B. Phys. Rev. Lett. 2002, 88, 173903.

(31) Kienberger, R.; Goulielmakis, E.; Uiberacker, M.; Baltuska, A.; Yakovlev, V.; Bammer, F.; Scrinzi, A.; Westerwalbesloh, T.; Kleineberg, U.; Heinzmann, U.; Drescher, M.; Krausz, F. Nature 2004, 427, 817-821.

(32) Kawada, Y.; Yasuda, T.; Takahashi, H. Opt. Lett. 2016, 41, 986.

(33) Hirori, H.; Doi, a.; Blanchard, F.; Tanaka, K. Appl. Phys. Lett. 2011, 98, 091106.

(34) Kawada, Y.; Yasuda, T.; Nakanishi, A.; Akiyama, K.; Hakamata, K.; Takahashi, H. Opt. Lett. 2014, 39, 2794.

(35) Simmons, J. G. J. Appl. Phys. 1963, 34, 1793-1803. 
(36) Wang, K.; Mittleman, D. M.; van der Valk, N. C. J.; Planken, P. C. M. Appl. Phys. Lett. 2004, 85,2715 .

(37) Chen, H.-T.; Kraatz, S.; Cho, G. C.; Kersting, R. Phys. Rev. Lett. 2004, 93, 267401.

(38) Atkin, J. M.; Berweger, S.; Jones, A. C.; Raschke, M. B. Adv. Phys. 2012, 61, 745-842.

(39) Eisele, M.; Cocker, T. L.; Huber, M. a.; Plankl, M.; Viti, L.; Ercolani, D.; Sorba, L.; Vitiello, M. S.; Huber, R. Nat. Photonics 2014, 8, 841-845.

(40) Ni, G. X.; Wang, L.; Goldflam, M. D.; Wagner, M.; Fei, Z.; McLeod, A. S.; Liu, M. K.;

Keilmann, F.; Özyilmaz, B.; Castro Neto, A. H.; Hone, J.; Fogler, M. M.; Basov, D. N. Nat. Photonics 2016, 10, 244-247.

(41) Ni, G. X.; McLeod, A. S.; Sun, Z.; Wang, L.; Xiong, L.; Post, K. W.; Sunku, S. S.; Jiang, B. Y.; Hone, J.; Dean, C. R.; Fogler, M. M.; Basov, D. N. Nature 2018, 557, 530-533.

(42) Yoshida, K.; Shibata, K.; Hirakawa, K. Phys. Rev. Lett. 2015, 115, 138302.

(43) Takeda, J.; Yoshioka, K.; Minami, Y.; Katayama, I. J. Phys. D. Appl. Phys. 2018, 51, 103001.

(44) Cocker, T. L.; Jelic, V.; Gupta, M.; Molesky, S. J.; Burgess, J. A. J.; Reyes, G. D. L.; Titova, L. V.; Tsui, Y. Y.; Freeman, M. R.; Hegmann, F. A. Nat. Photonics 2013, 7, 620-625.

(45) Savage, K. J.; Hawkeye, M. M.; Esteban, R.; Borisov, A. G.; Aizpurua, J.; Baumberg, J. J. Nature 2012, 491, 574-577.

(46) Scholl, J. A.; García-Etxarri, A.; Koh, A. L.; Dionne, J. A. Nano Lett. 2013, 13, 564-569. 
(47) Stockman, M. Phys. Rev. Lett. 2004, 93, 137404.

(48) Giugni, A.; Torre, B.; Toma, A.; Francardi, M.; Malerba, M.; Alabastri, A.; Proietti

Zaccaria, R.; Stockman, M. I.; Di Fabrizio, E. Nat. Nanotechnol. 2013, 8, 845-852. 
Insert Table of Contents Graphic and Synopsis Here.

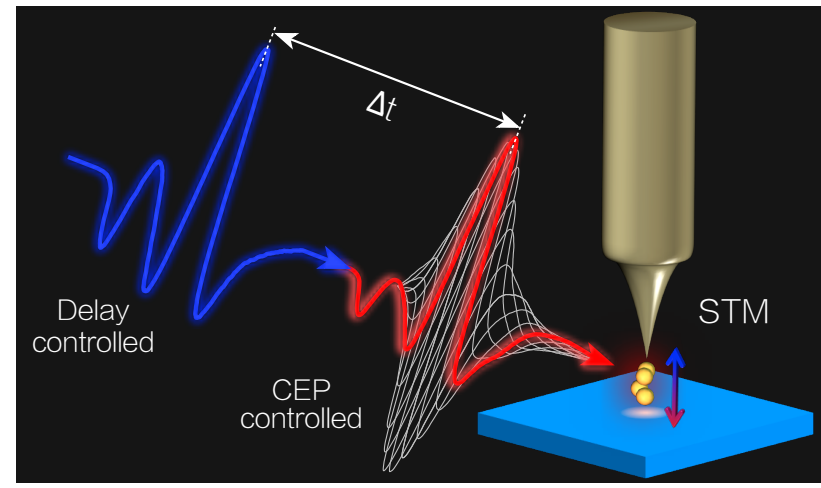

